\title{
Hemodynamic goal-directed therapy on Cytoreductive Surgery with Hyperthermic Intraperitoneal Chemotherapy
}

\author{
Esteve N, Ferrer A, Gómez G, Verd M, Melero C, Mora LC \\ Son Espases University Hospital \\ IdISPA, Health Research Institute of Palma
}

\section{Research Focus}

Cytoreductive Surgery (CRS) with Hyperthermic Intraperitoneal Chemotherapy (HIPEC) results in significant hemodynamic, metabolic and haematological changes ${ }^{1}$.

Much variation occurs during intraoperative management of these cases ${ }^{2}$. Controversy has risen on adequate monitoring, fluid therapy, metabolic changes and prevention and treatment of complications. We present the results of minimally invasive monitoring of Systolic Volume Variation (SVV) and hemodynamic goal-directed therapy.

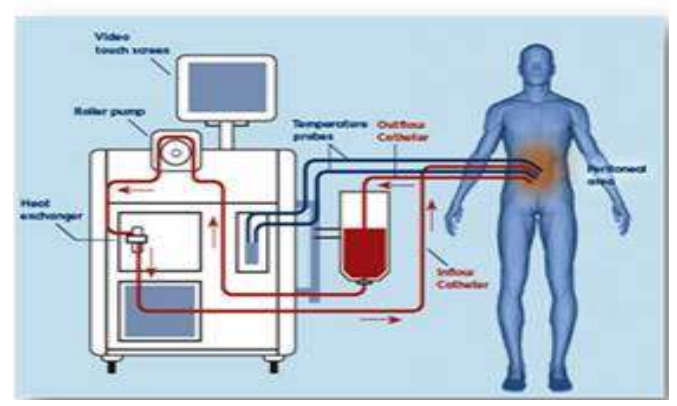

\section{Methods}

Prospective, descriptive study on patients with PC who underwent CRS with HIPEC between March 2014 and February 2015. We applied an anaesthesia protocol for monitoring and goals regarding hemodynamics, temperature control, fluid therapy, vasoactive drug use and extubation criteria.

We recorded the data at the beginning of anaesthesia, at various times during CRS and HIPEC and along the first three postoperative days.
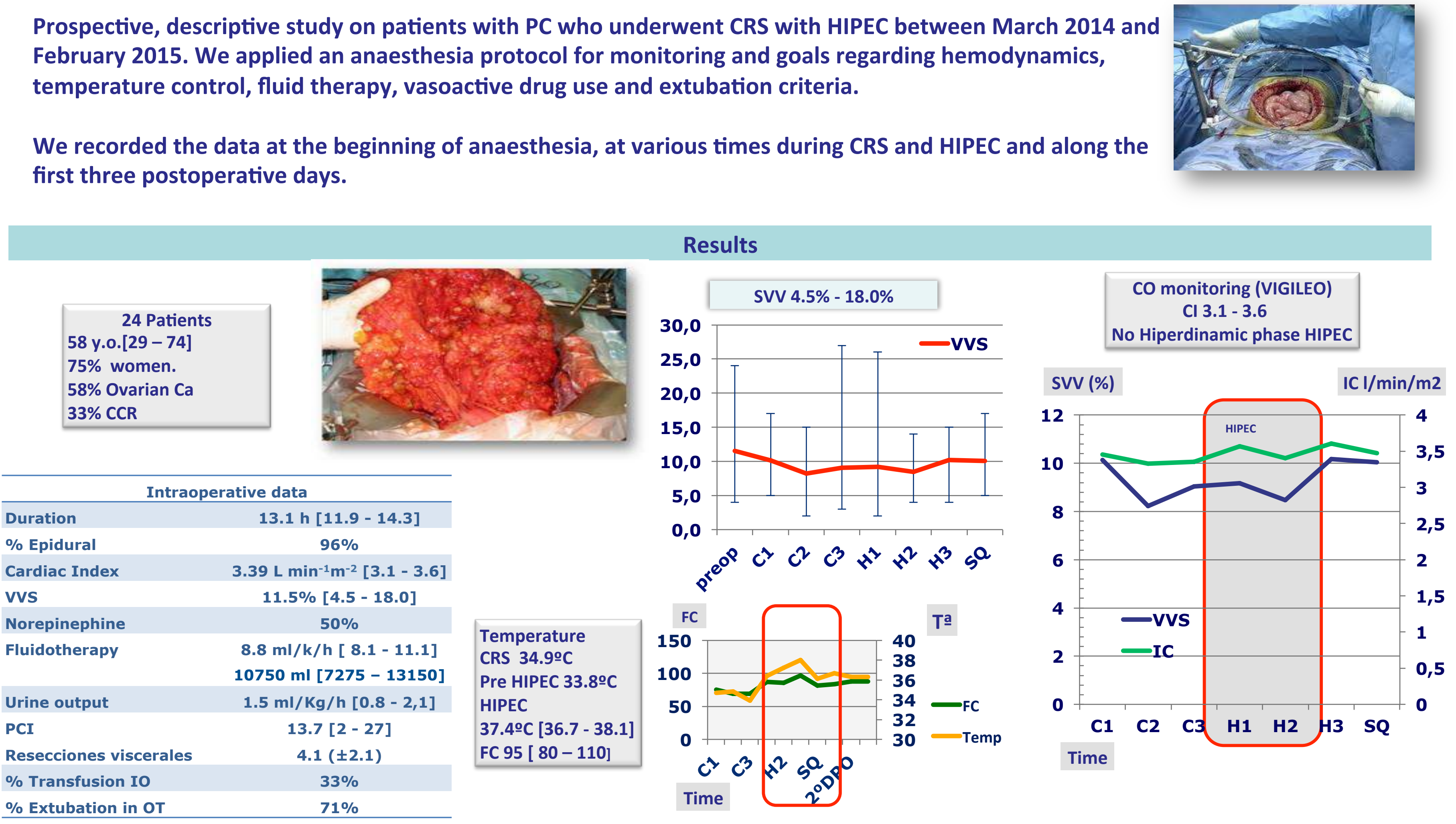

We included 24 patients. The main intraoperative data were: Intraperitoneal Carcinomatosis index 13.7 ( \pm 8.1 ), $96 \%$ received epidural analgesia, $92 \%$ underwent complete cytoreduction, $42 \%$ were transfused perioperatively, median fluid infusion was $8.8 \mathrm{ml} / \mathrm{kg} / \mathrm{h}$ [ 8.1 - 11.1] and urine output $1.5 \mathrm{ml} / \mathrm{kg} / \mathrm{h}[0.8-2,1]$. Cardiac index and SVV average intraoperatively were $3.39 \mathrm{~L} \mathrm{~min}^{-1} \mathrm{~m}^{-2}[3.1-3.6]$ and 11.5\% [4.5 - 18.0]. Maximum SVV was at the end of CRS and the beginning of HIPEC at $18 \%$ and $16.8 \%$ respectively.

Average oropharyngeal temperature during HIPEC was 37.4ㄷ [36.7 - 38.1]. Cardiac index and heart rate were stable through HIPEC. $\mathbf{5 0 \%}$ rate of norepinephrine use and $\mathbf{7 1 \%}$ of patients were extubated in the operating room. Postoperative morbidity was $\mathbf{2 5 \%}$ on severe complications (Clavien-Dindo grade III-IV) and no mortality was recorded.

\section{Conclusiones}

We found great inter-patient variability on fluid requirements. Monitoring SVV 3 allowed us to predict fluid therapy or vasoactive drug requirement during all stages of surgery and to tailor treatment towards greater hemodynamic stability.

The absence of a hyper-dynamic state during HIPEC as seen on other studies, can be accounted on the normovolemia accomplished on our series at the beginning of HIPEC.

1. Raue $W$ et al. Volume therapy and cardiocircular function during hyperthermic intraperitoneal chemotherapy. Eur Surg Res. 2009;43(4):365-72

2. Corbella $D$ et al. Anesthetic management of cytoreductive surgery and hyperthermic intraperitoneal chemotherapy procedures. World

J Obstet Gynecol 2013;10(4):129-36

3. Zhang $Z$ et al. Accuracy of stroke volume variation in predicting fluid responsiveness: a systematic review and meta-analysis. J Anesth 